\title{
A counting invariant for maps into spheres and for zero loci of sections of vector bundles
}

\author{
Panagiotis Konstantis
}

11th November 2019

\begin{abstract}
The set of unrestricted homotopy classes $\left[M, S^{n}\right]$ where $M$ is a closed and connected spin $(n+1)$ manifold is called the $n$-th cohomotopy group $\pi^{n}(M)$ of $M$. Moreover it is known that $\pi^{n}(M)=$ $H^{n}(M ; \mathbb{Z}) \oplus \mathbb{Z}_{2}$ by methods from homotopy theory. We will provide a geometrical description of the $\mathbb{Z}_{2}$ part in $\pi^{n}(M)$ analogous to Pontryagin's computation of the stable homotopy group $\pi_{n+1}\left(S^{n}\right)$. This $\mathbb{Z}_{2}$ number can be computed by counting embedded circles in $M$ with a certain framing of their normal bundle. This is a similar result to the mod 2 degree theorem for maps $M \rightarrow S^{n+1}$.

Finally we will observe that the zero locus of a section in an oriented rank $n$ vector bundle $E \rightarrow M$ defines an element in $\pi^{n}(M)$ and it turns out that the $\mathbb{Z}_{2}$ part is an invariant of the isomorphism class of $E$. At the end we show, that if the Euler class of $E$ vanishes this $\mathbb{Z}_{2}$ invariant is the final obstruction to the existence of a nowhere vanishing section.
\end{abstract}

\section{Introduction}

Pontryagin computed in [14] the (stable) homotopy group $\pi_{n+1}\left(S^{n}\right)(n \geq 3)$ by using differential topology. Let us describe briefly his construction, since this paper will generalize his idea.

Pontryagin showed that $\pi_{n+1}\left(S^{n}\right)$ is isomorphic to the bordism group of closed 1-dimensional submanifolds of $\mathbb{R}^{n+1}$ furnished with a framing on its normal bundle (a framing is a homotopy class of trivializations, see section 2). We denote this bordism group by $\Omega_{1}^{\mathrm{fr}}\left(\mathbb{R}^{n+1}\right)$. Let $(C, \varphi)$ be a representative of an element of $\Omega_{1}^{\mathrm{fr}}\left(\mathbb{R}^{n+1}\right)$, i.e. $C$ is a union of embedded circles in $\mathbb{R}^{n+1}$ and there are maps $\varphi_{1}, \ldots, \varphi_{n}: C \rightarrow \mathbb{R}^{n+1}$ such that $\left(\varphi_{1}(x), \ldots, \varphi_{n}(x)\right)$ is a basis of $\nu(C)_{x}$ for every $x \in C$. Let $\varphi_{n+1}$ be a trivialization of the tangent bundle of $C$. Then $\left(\varphi_{1}(x), \ldots, \varphi_{n+1}(x)\right)$ is a basis of $\mathbb{R}^{n+1}$ for every $x \in C$. Without loss of generality we may assume that $\varphi_{1}, \ldots, \varphi_{n+1}$ is pointwise an orthonormal basis. If $\left(e_{1}, \ldots, e_{n+1}\right)$ denotes the standard basis of $\mathbb{R}^{n+1}$ then consider the map $A=\left(a_{i j}\right): C \rightarrow \operatorname{SO}(n+1)$ such that

$$
\varphi_{i}(x)=\sum_{j=1}^{n+1} a_{i j}(x) e_{j}
$$

for $x \in C$. Let $\pi_{1}(\mathrm{SO}(n+1))$ be identified with $\mathbb{Z}_{2}$, then Pontryagin defines [14, Theorem 20]

$$
\delta(C, \varphi):=[A]+(n(C) \bmod 2)
$$

where $[A]$ denotes the homotopy class of $A$ in $\pi_{1}(\mathrm{SO}(n+1))$ and $n(C)$ is the number of connected components of $S$. He showed that $\delta$ is well-defined on $\Omega_{1}^{\mathrm{fr}}\left(\mathbb{R}^{n+1}\right)$ and is an isomorphism of groups.

From a different point of view, one may consider his computation not as a computation of a homotopy group of $S^{n}$ but rather of a cohomotopy group of $S^{n+1}$. If $X$ is a CW space then the cohomotopy set of $X$ is defined as the set of (unrestricted) homotopy classes $\pi^{n}(X):=\left[X, S^{n}\right]$, cf. [3,15]. The set $\pi^{n}(X)$ for $X$ a finite $\mathrm{CW}$ complex of dimension $n+1$ carries naturally a group structure, which is described in the beginning of section 4. Steenrod showed [16, Theorem 28.1, p. 318] that $\pi^{n}(X)$ fits into a short exact sequence

$$
0 \longrightarrow H^{n+1}\left(X ; \mathbb{Z}_{2}\right) / \mathrm{Sq}^{2} \mu\left(H^{n-1}(X ; \mathbb{Z})\right) \longrightarrow \pi^{n}(X) \longrightarrow H^{n}(X ; \mathbb{Z}) \longrightarrow 0,
$$


where $\mu: H^{*}(X ; \mathbb{Z}) \rightarrow H^{*}\left(X ; \mathbb{Z}_{2}\right)$ is the $\bmod 2$ reduction homomorphism. Here the surjective map is the Hurewicz homomorphism which assigns to every $f \in \pi^{n}(X)$ the cohomology class $f^{*}(\sigma) \in H^{n}(X ; \mathbb{Z})$ where $\sigma \in H^{n}\left(S^{n}, \mathbb{Z}\right)$ is a fixed generator.

Moreover using methods of Larmore and Thomas [10] Taylor showed in [17, Theorem 6.2, Example $6.3]$ that the short exact sequence splits, provided the images of $\mathrm{Sq}^{2}: H^{n-1}\left(X ; \mathbb{Z}_{2}\right) \rightarrow H^{n+1}\left(X ; \mathbb{Z}_{2}\right)$ and $\mathrm{Sq}^{2} \circ \mu: H^{n-1}(X ; \mathbb{Z}) \rightarrow H^{n+1}\left(X ; \mathbb{Z}_{2}\right)$ coincide.

If $X=M$ is a manifold then the second $W u$ class [21] is equal to the second Stiefel-Whitney class $w_{2}(M)$, hence $\operatorname{Sq}^{2}(x)=w_{2}(M) \smile x$ for $x \in H^{n-1}\left(M ; \mathbb{Z}_{2}\right)$. Therefore if $M$ is spin then $\pi^{n}(M)$ fits into the exact sequence

$$
0 \longrightarrow \mathbb{Z}_{2} \longrightarrow \pi^{n}(M) \longrightarrow H^{n}(M ; \mathbb{Z}) \longrightarrow 0 .
$$

and (ST) splits by [17, Example 6.3] thus

$$
\pi^{n}(M) \cong H^{n}(M ; \mathbb{Z}) \oplus \mathbb{Z}_{2}
$$

as abelian groups. However the splitting map is constructed in a purely homotopy theoretic setting and an aim of this article is to provide a geometric description in case $M$ is a spin manifold.

This splitting map $\kappa: \pi^{n}(M) \rightarrow \mathbb{Z}_{2}$ (see Definition 3.8) for (ST) will be constructed similarly to Pontryagin's invariant $\delta$ from above. An important ingredient in Pontryagin's construction was the canonical background framing by the standard basis of $\mathbb{R}^{n+1}$, which allowed him to define the map $A: S \rightarrow \mathrm{SO}(n+1)$. In general if we replace $S^{n+1}$ or $\mathbb{R}^{n+1}$ by $M$, this background framing is not available any more. But this can be circumvented by using the spin structure of $M$, since over a circle every vector bundle with a spin structure defines a certain framing, cf. Lemma 3.1. Section 4 is devoted to determine geometrically the kernel of the Hurewicz map $\pi^{n}(M) \rightarrow H^{n}(M ; \mathbb{Z})$. Finally we show that the splitting map possesses a naturality property, cf. Proposition 4.3 and that for a map $f: M \rightarrow S^{n}$ the number $\kappa(f)$ can be described by a counting formula, cf. Corollary 4.4. This is an analogous result to the mod 2 Hopf theorem, see [13,4]. It should be mentioned that in [7] the authors discuss the case $n=3$ and in [9] a similar construction of a $\mathbb{Z}_{2}$ invariant was used to classify quaternionic line bundles over closed spin 5 -manifolds.

In Section 5 we will apply the results of Sections 3 and 4 to the theory of vector bundles. Suppose $E \rightarrow M$ is a oriented vector bundle of rank $n$ over a closed spin $(n+1)$-manifold $M$. Then any section of $E$ which is transverse to the zero section defines by means of its zero locus an element of $\Omega_{1}^{\text {fr }}(M)$ and this element is independent of the transverse section. Thus using $\kappa$ one defines an invariant $\kappa(E) \in \Omega_{1}^{\mathrm{fr}}$ of the isomorphism class of the bundle $E \rightarrow M$. In Theorem 5.5 it is shown, that $\kappa(E)$ can be regarded as the secondary obstruction to the existence of a nowhere vanishing section. As an application we provide in Example 5.6 a simple proof of the well-known fact, that the maximal number of linear independent vector fields on $S^{4 k+1}$ is equal to 1 . Finally we show that $\pi^{n}(M)$ can be mapped injectively into the set of isomorphism classes of oriented rank $n$ vector bundles over spin $(n+1)$-manifolds for $n=4$ and $n=8$, cf. Proposition 5.8.

\section{Acknowledgements}

The author would like to thank S. Carmeli and M. Miller for helpful discussions in [8].

\section{Preliminaries}

If not otherwise stated we denote by $M$ an $(n+1)$-dimensional oriented, closed and connected manifold, where $n \geq 3$. Let $N$ be a arbitrary manifold and $E \rightarrow N$ a trivial vector bundle over $N$ of rank $r$. A trivialization of $E \rightarrow N$ are $r$ sections $s_{1}, \ldots, s_{r}: N \rightarrow E$, such that $\left(s_{1}(q), \ldots, s_{r}(q)\right)$ is a basis of the fiber $E_{q}$ for all $q \in N$. A framing $\varphi$ of $E \rightarrow N$ is a homotopy class of trivializations.

We recall now the notion of bordism classes of normally framed submanifold in $M$ of dimension $k$ (cf. $[13,7]$ ). Let $C$ be a $k$-dimensional closed submanifold of $M$. We say that $C$ is normally framed if the normal bundle of $C$ is trivial and possesses a framing $\varphi$. Two such normally framed submanifolds $\left(C_{0}, \varphi_{0}\right)$ and $\left(C_{1}, \varphi_{1}\right)$ are framed bordant if there is a $(k+1)$-dimensional submanifold $\Sigma \subset M \times[0,1]$ such that 
(a) $\partial \Sigma \cap(M \times i)=C_{i}$ for $i=0,1$,

(b) $\partial \Sigma=C_{0} \cup C_{1}$,

(c) $\Sigma$ is normally framed in $M \times[0,1]$ such that the framing restricted to the $\partial \Sigma \cap(M \times i)$ coincides with $\varphi_{i}$.

To be framed bordant is an equivalence relation and the set of equivalence classes is called the bordism classes of normally framed $k$-dimensional submanifolds denoted by $\Omega_{k}^{\mathrm{fr}}(M)$. If $(C, \varphi)$ is a normally framed submanifold then we denote by $[C, \varphi]$ its bordism class in $\Omega_{k}^{\mathrm{fr}}(M)$.

The Pontryagin-Thom map provides a bijection between $\pi^{n+1-k}(M)$ and $\Omega_{k}^{\mathrm{fr}}(M)$ as follows (cf. [13, 7]): Let $f: M \rightarrow S^{n+1-k}$ represents an element of $\pi^{n+1-k}(M)$. Choose a regular value $x_{0} \in S^{n+1-k}$ and set $C_{x_{0}}:=f^{-1}\left(x_{0}\right)$. Moreover choosing a basis of the tangent space $T_{x_{0}} S^{n+1-k}$ endows the normal bundle with a framing $\varphi_{x_{0}}$ by means of the derivative of $f$. The bordism class $\left[C_{x_{0}}, \varphi_{x_{0}}\right] \in \Omega_{k}^{\mathrm{fr}}(M)$ is well defined and the map

$$
\pi^{n+1-k}(M) \longrightarrow \Omega_{k}^{\mathrm{fr}}(M), \quad[f] \mapsto\left[C_{x_{0}}, \varphi_{x_{0}}\right] .
$$

is a bijection, see [13, Theorem $\mathrm{B}$ and $\mathrm{A}]$.

A stable framing of a real vector bundle $E \rightarrow C$ of rank $r$ is an equivalence class of trivializations of

$$
E \oplus \varepsilon^{l}
$$

for some $l \in \mathbb{N}$ where two trivializations

$$
\tau_{1}: E \oplus \varepsilon^{l_{1}} \rightarrow \varepsilon^{r+l_{1}} \quad \text { and } \quad \tau_{2}: E \oplus \varepsilon^{l_{2}} \rightarrow \varepsilon^{r+l_{2}},
$$

are considered to be equivalent if there exists some $L>l_{1}, l_{2}$ such that the isomorphisms

$$
\tau_{1} \oplus \mathrm{id}: E \oplus \varepsilon^{l_{1}} \oplus \varepsilon^{L-l_{1}} \rightarrow \varepsilon^{L+r}
$$

and

$$
\tau_{2} \oplus \mathrm{id}: E \oplus \varepsilon^{l_{2}} \oplus \varepsilon^{L-l_{2}} \rightarrow \varepsilon^{L+r}
$$

are homotopic, cf. [4, Section 8.3]. If $E$ is the tangent bundle of $C$, then a stable framing of $T C$ is called a stable tangential framing. If $E$ is the normal bundle of an embedding of $C$ into a sphere of big dimension, then we call a stable framing a stable normal framing.

We define $\Omega_{k}^{\mathrm{fr}}$ to be the bordism classes of stably (tangential) framed manifolds. More precisely two stably framed manifolds $\left(C_{0}, \varphi_{0}\right)$ and $\left(C_{1}, \varphi_{1}\right)$ where $\varphi_{i}: T C_{i} \oplus \varepsilon^{l} \rightarrow \varepsilon^{k+l}$ is an isomorphism are equivalent if there is a bordism $\Sigma$ between $C_{0}$ and $C_{1}$ such that the tangent bundle of $\Sigma$ possesses a stable framing and the restriction on $C_{0}$ and $C_{1}$ coincides with the framing $\varphi_{0}$ and $\varphi_{1}$ respectively. Note that $\Omega_{k}^{\mathrm{fr}}$ is isomorphic to $\pi_{k}^{S}$, the $k$-stable homotopy group of spheres (cf. [4, Theorem 8.17]) and by the Pontryagin-Thom construction we have $\Omega_{k}^{\mathrm{fr}}=\lim _{l} \Omega_{k}^{\mathrm{fr}}\left(S^{l}\right)$ where we use the equatorial embeddings $S^{l_{1}} \hookrightarrow S^{l_{2}}$ if $l_{1}<l_{2}$ to construct well-defined maps $\overrightarrow{\Omega_{k}^{\mathrm{fr}}}\left(S^{l_{1}}\right) \rightarrow \Omega_{k}^{\mathrm{fr}}\left(S^{l_{2}}\right)$.

For this article the case $k=1$ will be of importance. In this case we have $\Omega_{1}^{\text {fr }} \cong \pi_{1}^{S} \cong \mathbb{Z}_{2}$. Consider a connected and closed 1-dimensional manifold $S_{0}$ and stable tangential framing

$$
\varphi_{0}: T S_{0} \oplus \varepsilon^{n} \stackrel{\sim}{\longrightarrow} \varepsilon^{n+1} .
$$

From the discussion above, $\left(S_{0}, \varphi_{0}\right)$ defines a class in $\Omega_{1}^{\mathrm{fr}}$ and can be realized as follows: Consider $S_{0}=\left\{\left(x_{1}, \ldots, x_{n+1}\right) \in \mathbb{R}^{n+1}: x_{1}^{2}+x_{2}^{2}=1, x_{i}=0, i=3, \ldots, n+1\right\}$. Denote $e_{1}, \ldots, e_{n+1}$ the canonical basis of $\mathbb{R}^{n}$ and $E_{i}(x)=e_{i}$ for $x \in \mathbb{R}^{n+1}$ the constant vector fields on $\mathbb{R}^{n+1}$. Moreover let $V(x)=x$ for $x \in \mathbb{R}^{n}$. The normal bundle $\nu\left(S_{0}\right)$ of $S_{0}$ is trivialized by $V, E_{3}, \ldots, E_{n+1}$ restricted to $S_{0}$. Using this normal framing we obtain a stable framing

$$
\left.T S_{0} \oplus \varepsilon^{n} \cong T S_{0} \oplus \nu\left(S_{0}\right) \cong\left(T \mathbb{R}^{n+1}\right)\right|_{S_{0}} \cong \varepsilon^{n+1}
$$

where the latter framing is induced by $E_{1}, \ldots, E_{n+1}$. Hence this defines an element in $\Omega_{1}^{\mathrm{fr}}\left(S^{n+1}\right)$ which represents the framed null bordism, since the framing of $\nu\left(S_{0}\right)$ can be extended to a properly embedded stably framed disc in $S^{n+1} \times[0,1]$. Clearly the non-trivial element of $\Omega_{1}^{\mathrm{fr}}\left(S^{n+1}\right)$ can be represented by twisting the normal framing $E_{3}, \ldots, E_{n+1}$ with a map $S_{0} \rightarrow \mathrm{SO}(n)$ such that its homotopy class in 
$\pi_{1}(\mathrm{SO}(n)) \cong \mathbb{Z}_{2}$ is not zero. Every stable tangential framing of a closed and connected 1-dimensional manifold can be obtain in this way.

If $E \rightarrow N$ is an oriented vector bundle over a manifold $N$, then we say that $E$ is spinnable if the second Stiefel-Whitney class $w_{2}(E)$ is zero. This means that $E$ can carry a spin structure, that is a lift of the classifying map $N \rightarrow B \mathrm{SO}(n)$ to a map $N \rightarrow B \operatorname{Spin}(n)$ in the fibration $K\left(\mathbb{Z}_{2}, 1\right) \rightarrow B \operatorname{Spin}(n) \rightarrow B \operatorname{SO}(n)$. Consequently $E$ is a spin bundle is it spinnable and a spin structure is fixed. If a spin structure is fixed on $E \rightarrow N$ then any other spin structure is in $1: 1$ correspondence with elements in $H^{1}\left(N ; \mathbb{Z}_{2}\right)$.

We write $F(N)$ for the frame bundle of a manifold $N$. If $V \subset N$ is a submanifold such that its normal bundle is framed then we obtain an embedding $F(V) \subset F(N)$. Thus a spin structure on $N$ induces a spin structure on $V$, cf. [12]. In particular if $V$ is the boundary of a spin manifold $N$, then $V$ inherits a spin structure from $N$. Finally if $E \rightarrow N$ is a vector bundle with a spin structure and $V \subset N$ a submanifold, then clearly $\left.E\right|_{V} \rightarrow V$ also inherits a spin structure from $E \rightarrow N$.

Let $E \rightarrow S^{1}$ be a spinnable vector bundle of rank $r \geq 3$ over the unit circle $S^{1}$. Then $E$ has exactly two non-isomorphic spin structures. Clearly $E \rightarrow S^{1}$ can be extended to $E \rightarrow D^{2}$, where $D^{2}$ denotes the closed unit disc in $\mathbb{R}^{2}$. Since $D^{2}$ is contractible $E \rightarrow D^{2}$ admits a unique spin structure. Restricting this structure to the boundary of $D^{2}$ gives a spin structure on $E \rightarrow S^{1}$, which will be called the standard spin structure. The other should be called the non-standard spin structure. In other words, the standard spin structure on $E \rightarrow S^{1}$ can be extended to $D^{2}$, the non-standard not.

\section{The index of framed circles}

We define in this section the key invariant of this article. For its construction the following basic lemma is the crucial observation.

Lemma 3.1. Let $E \rightarrow S^{1}$ be a spinnable vector bundle of rank $\geq 3$. Then $E$ is isomorphic to the trivial bundle and a choice of a spin structure on $E$ determines a framing on $E$.

Proof. $E$ is isomorphic to the trivial bundles since it is an orientable vector bundle over a circle. Fix a spin structure on $E$, i.e. let $F^{\prime}(E)$ be a $\operatorname{Spin}(n)$-principal bundle over $S^{1}$ which is a two-sheeted cover over the frame bundle $F(E)$ of $E$. Let $\pi: F^{\prime}(E) \rightarrow F(E)$ be the projection which is equivariant with respect to the two-sheeted covering $\mathbf{S p i n}(n) \rightarrow \mathbf{S O}(n)$. Clearly $F^{\prime}(E)$ is the trivial $\mathbf{S p i n}(n)$-principal bundle over $S^{1}$ and denote by $\sigma: S^{1} \rightarrow F^{\prime}(E)$ a global section. Then $\pi \circ \sigma$ is a global section of $F(E)$ hence a trivialization of $E \rightarrow S^{1}$. Any other such global section $\widetilde{\sigma}: S^{1} \rightarrow E$ differs from $\sigma$ by a map $\varphi: S^{1} \rightarrow \operatorname{Spin}(n)$. Since $\pi_{1}(\operatorname{Spin}(n))=1$ the map $\varphi$ has to be null-homotopic which means that the two trivializations $\pi \circ \sigma$ and $\pi \circ \widetilde{\sigma}$ have to be homotopic, thus they define the same framing on $E$.

In the same way one proves

Corollary 3.2. Let $\Sigma$ be a 1-dimensional $C W$-complex (not necessarily connected) and $E \rightarrow \Sigma$ a vector bundle of rank $\geq 3$ endowed with a spin structure. Then $E$ is isomorphic to the trivial bundle and the spin structure induces a framing on $E$.

Definition 3.3. Let $E \rightarrow S^{1}$ be a spinnable vector bundle. The framing induced by the standard spin structure on $E$ is called the standard framing and from then non-standard spin structure the non-standard framing.

Example 3.4. The spheres $S^{n+1}$ admit a unique spin structure which can be constructed as mentioned in the preliminaries, i.e. $S^{n+1}$ is the boundary of the closed unit ball $D^{n+2}$ in $\mathbb{R}^{n+2}$ which admits a unique spin structure.

Let $S_{0} \in S^{n+1}$ be the intersection of a 2-dimensional linear subspace $W \subset \mathbb{R}^{n+2}$ with $S^{n+1}$ and denote by $D_{0}^{2}=W \cap D^{n+2}$. Thus after Lemma $\left.3.1 T S^{n+1}\right|_{S_{0}}$ inherits a framing from the spin structure. Denote by $\varphi_{1}, \ldots, \varphi_{n+1}$ a trivialization of this framing, then the framing

$$
\bar{\varphi}: S_{0} \rightarrow \mathrm{SO}(n+2), \quad x \mapsto\left(x, \varphi_{1}(x), \ldots, \varphi_{n+1}(x)\right)
$$

must be null homotopic in $\mathrm{SO}(n+2)$ by the definition of the spin structures of $S^{n+1}$ and $\left.T S^{n+1}\right|_{S_{0}}($ such that it lifts to $\operatorname{Spin}(n+2))$. Thus $\bar{\varphi}$ must be homotopic the constant framing $x \mapsto\left(e_{1}, \ldots, e_{n+2}\right)$, where $e_{1}, \ldots, e_{n+2}$ denotes the canonical basis of $\mathbb{R}^{n+2}$. In particular this means, that $\left.T S^{n+1}\right|_{S_{0}}$ inherits the standard framing from the spin structure of $S^{n+1}$. 
$\Omega_{1}^{\mathrm{fr}}(M)$ possesses a group structure which can be expressed as follows: Having two 1-dimensional closed submanifolds $C$ and $C^{\prime}$ of $M$ which are normally framed, then they are framed bordant in $M$ to framed submanifolds $\tilde{C}$ and $\tilde{C}^{\prime}$ whose intersection is empty. Taking the equivalence class of the disjoint union $\tilde{C} \cup \tilde{C}^{\prime}$ with the respective framings yields an abelian group structure on $\Omega_{1}^{\mathrm{fr}}(M)$, cf. [13, Problem 17 and p. 50].

Next, we construct a homomorphism $\kappa: \Omega_{1}^{\mathrm{fr}}(M) \rightarrow \Omega_{1}^{\mathrm{fr}}$, where $\Omega_{1}^{\mathrm{fr}}$ is the bordism group of stably framed 1-dimensional closed manifolds. Therefore let $\left(C, \varphi_{C}\right)$ be a closed submanifold of dimension 1 , such that its normal bundle $\nu(C)$ is framed by $\varphi_{C}$ (thus representing an element in $\Omega_{1}^{\text {fr }}(M)$ ). From Lemma 3.1 the bundle $\left.T M\right|_{C}$ inherits a framing $\varphi_{\sigma}$ from the spin structure of $M$. Using also the framing of $\varphi_{C}$ we obtain a stable tangential framing

$$
\left.\varepsilon^{n+1} \cong T M\right|_{C} \cong T C \oplus \nu(C) \cong T C \oplus \varepsilon^{n}
$$

which we denote by $\varphi_{\text {st }}$.

Proposition 3.5. The bordism class $\left[C, \varphi_{s t}\right] \in \Omega_{1}^{f r}$ depends only on the bordism class of $\left[C, \varphi_{C}\right] \in$ $\Omega_{1}^{f r}(M)$.

Proof. Let $\left(C^{\prime}, \varphi_{C^{\prime}}\right)$ be another normally framed closed 1-dimensional submanifold framed bordant to $\left(C, \varphi_{C}\right)$. Thus there is a bordism $\Sigma \subset M \times I$ between $C$ and $C^{\prime}$ such that the normal bundle of $\Sigma$ in $M \times I$ possess a framing $\varphi_{\Sigma}$. By definition restricting $\varphi_{\Sigma}$ to $C$ and $C^{\prime}$ yields $\varphi_{C}$ and $\varphi_{C^{\prime}}$ respectively. Since $\Sigma$ is homotopy equivalent to a 1-dimensional CW-complex and since $M \times I$ inherits a unique spin structure from $M$ we obtain a framing $\varphi_{\Sigma, \sigma}$ on $\left.T(M \times I)\right|_{\Sigma}$. Of course the framings $\varphi_{\Sigma, \sigma}$ restricted to $C$ and $C^{\prime}$ are just the framings $\varphi_{\sigma}$ and $\varphi_{\sigma}^{\prime}$ respectively (i.e. induced by the spin structure of $\left.T M\right|_{C}$ and $\left.\left.T M\right|_{C^{\prime}}\right)$. Since

$$
\left.T(M \times I)\right|_{\Sigma} \cong T \Sigma \oplus \nu(\Sigma)
$$

the framings $\varphi_{\Sigma, \sigma}$ and $\varphi_{\Sigma}$ determine a stable framing $\varphi_{\Sigma, \mathrm{st}}$ of $T \Sigma$. Then $\left(\Sigma, \varphi_{\Sigma, \mathrm{st}}\right)$ is stably framed bordism between $\left(C, \varphi_{\text {st }}\right)$ and $\left(C^{\prime}, \varphi_{\text {st }}^{\prime}\right)$.

Remark 3.6. As described above, the group structure of $\Omega_{1}^{\mathrm{fr}}(M)$ is given by disjoint union of submanifolds and their respective normal framings. Let $(C, \varphi)$ be a framed 1-dimensional closed submanifold of $M$ and denote by $C=S_{1} \cup \ldots \cup S_{k}$ the connected components of $C$. We may assume that the union is always disjoint. Thus $S_{i}$ is an embedded circle and $\varphi_{i}:=\left.\varphi\right|_{S_{i}}$ a normal framing of $S_{i}$. Consequently we have

$$
[C, \varphi]=\sum_{i=1}^{k}\left[S_{i}, \varphi_{i}\right]
$$

Definition 3.7. Let $S \subset M$ be an embedded circle and $\varphi$ a framing of $\nu(S)$. We call the bordism class $[S, \varphi] \in \Omega_{1}^{\mathrm{fr}}(M)$ a framed circle of $M$. The corresponding stable class $\left[S, \varphi_{\mathrm{st}}\right] \in \Omega_{1}^{\mathrm{fr}}$ will be called the the index of $[S, \varphi]$ (with respect to the spin structure of $M$ ) and will be denoted by $\operatorname{ind}(S, \varphi)$.

Definition 3.8. Let $M$ be an $(n+1)$-dimensional closed spin manifold. Then we define a map

$$
\kappa: \Omega_{1}^{\mathrm{fr}}(M) \rightarrow \Omega_{1}^{\mathrm{fr}}, \quad[C, \varphi] \mapsto \kappa([C, \varphi]):=\sum_{\substack{S \subset C, S \text { connected }}} \operatorname{ind}\left(S,\left.\varphi\right|_{S}\right)=\left[C, \varphi_{\mathrm{st}}\right] .
$$

We call $\kappa$ the degree map of $M$ with respect to the chosen spin structure.

Remark 3.9. It is clear from the construction that $\kappa$ is a homomorphism.

Examples 3.10. (a) The spheres $S^{n+1}$ admit a unique spin structure which is induced by the closed $(n+2)$-dimensional disc $D^{n+2} \subset \mathbb{R}^{n+2}$, cf. [12].

Let $S_{0}$ be the intersection of $S^{n+1}$ with a 2-dimensional linear subspace $W$ of $\mathbb{R}^{n+2}$. We argued in Example 3.4 that $\left.T S^{n+1}\right|_{S_{0}}$ inherits the standard framing.

Choose the standard framing $\varphi_{0}$ on $\nu\left(S_{0}\right)$, then

$$
\kappa\left(\left[S_{0}, \varphi_{0}\right]\right)=0 .
$$

Consequently the non-standard framing $\varphi_{1}$ of $\nu\left(S_{0}\right)$ yields

$$
\kappa\left(\left[S_{0}, \varphi_{1}\right]\right) \neq 0 .
$$


(b) Let $N$ be a closed, simply connected, spin manifold of dimension $n$. Then $M:=S^{1} \times N$ admits two different spin structures since $H^{1}\left(S^{1} \times N ; \mathbb{Z}_{2}\right) \cong H^{1}\left(S^{1} ; \mathbb{Z}_{2}\right) \cong \mathbb{Z}_{2}$. $M$ is the boundary of $D^{2} \times N$ which has up to isomorphism a unique spin structure. The two different spin structures on $M$ can be described as follows: One can be extended from $M$ to $D^{2} \times N$ and the other not. We call the latter one the standard spin structure and the former one the non-standard spin structure of $S^{1} \times N$.

For $q_{0} \in N$ consider the circle $S_{0}:=S^{1} \times q_{0} \subset S^{1} \times N$. Clearly we have a canonical isomorphism

$$
\nu\left(S_{0}\right) \cong S_{0} \times T_{q_{0}} N
$$

Thus choosing a basis in $T_{q_{0}} N$ gives a framing $\varphi_{0}$ on $\nu\left(S_{0}\right)$ which extends to a framing of $\left(D^{2} \times\right.$ $\left.q_{0}\right) \times T_{q_{0}} N$. Thus we have

$$
\kappa_{0}\left(\left[S_{0}, \varphi_{0}\right]\right)=0
$$

for the standard spin structure and

$$
\kappa_{1}\left(\left[S_{0}, \varphi_{0}\right]\right) \neq 0
$$

for the non-standard spin structure.

For $q_{1} \in N$ with $q_{0} \neq q_{1}$ we consider $C=S^{1} \times q_{0} \cup S^{1} \times q_{1}$ with fixed normal framing on $S^{1} \times q_{i}$ which gives a framing $\varphi$ on $C$. Then $\kappa([C, \varphi])$ is independent of the chosen spin structure of $M$.

This shows that in general $\kappa$ will depend on the spin structure. The next proposition will show how it depends from it.

Suppose $C \subset M$ is a closed 1-dimensional submanifold. Then $C$ defines a $\mathbb{Z}_{2}$ fundamental homology class $[C] \in H_{1}\left(M ; \mathbb{Z}_{2}\right)$. We denote by $w(C) \in H^{n}\left(M ; \mathbb{Z}_{2}\right)$ the cohomology class which is the Poincaré dual of $[C]$.

Proposition 3.11. Fix a spin structure $\sigma$ on $M$ and denote by $\kappa$ the degree map of $M$ with respect to the chosen spin structure. Choose another spin structure of $M$, which is represented by $\alpha \in H^{1}\left(M ; \mathbb{Z}_{2}\right)$ and denote by $\kappa^{\alpha}$ the corresponding degree map. Then we have

$$
\kappa([C, \varphi])=\kappa_{\alpha}([C, \varphi])+\delta(\alpha \smile w(C)),
$$

where $\delta: H^{n+1}\left(M ; \mathbb{Z}_{2}\right) \rightarrow \Omega_{1}^{f r}$ is the unique isomorphism. Thus if $w(C) \smile \alpha=0$ then $\kappa([C, \varphi])=$ $\kappa^{\alpha}([C, \varphi])$.

Proof. Assume first that $(S, \varphi)$ is a framed circle and $i: S \rightarrow M$ is the inclusion. The spin structure $\sigma$ induces a spin structure on $\left.T M\right|_{S}=i^{*}(T M)$ and the spin structure induced by $\alpha$ is represented by $i^{*}(\alpha) \in H^{1}\left(S ; \mathbb{Z}_{2}\right)$. Of course $\left.T M\right|_{S}$ can have at most two different spin structures. From the definition of the index we have

$$
\operatorname{ind}(S, \varphi)=\operatorname{ind}_{\alpha}(S, \varphi)+\delta\left(i^{*}(\alpha)\right)
$$

where ind is defined by $\sigma$, ind $\alpha$ by $\alpha$ and $\delta: H^{1}\left(S ; \mathbb{Z}_{2}\right) \rightarrow \Omega_{1}^{\text {fr }}$ the unique isomorphism.

Let $[S] \in H_{1}\left(S ; \mathbb{Z}_{2}\right)$ be the $\mathbb{Z}_{2}$ fundamental class of $S$, then $i^{*}(\alpha) \frown[S] \in H_{0}\left(S ; \mathbb{Z}_{2}\right)$, which is mapped under $i_{*}$ to $\alpha \frown i_{*}([S]) \in H_{0}\left(M ; \mathbb{Z}_{2}\right)$. Let $[M] \in H_{n}\left(M ; \mathbb{Z}_{2}\right)$ denote the $\mathbb{Z}_{2}$ fundamental class of $M$. Then we compute

$$
\alpha \frown i_{*}([S])=\alpha \frown(w(S) \frown[M])=(\alpha \smile w(S)) \frown[M],
$$

where we used that $i_{*}([S])$ is Poincaré dual to $w(S)$. Since $\cdot \frown[S]$ and $\cdot \frown[M]$ are isomorphisms by Poincaré duality and $i_{*}: H_{0}\left(S ; \mathbb{Z}_{2}\right) \rightarrow H_{0}\left(M ; \mathbb{Z}_{2}\right)$ is also an isomorphisms because $S$ and $M$ are connected we infer

$$
\operatorname{ind}(S, \varphi)=\operatorname{ind}_{\alpha}(S, \varphi)+\delta(\alpha \smile w(S))
$$

where now $\delta: H^{n+1}\left(M ; \mathbb{Z}_{2}\right) \rightarrow \Omega_{1}^{\mathrm{fr}}$ is again the unique isomorphism.

Consider now $(C, \varphi)$ with the disjoint union $C=S_{1} \cup \ldots S_{k}$ and $\varphi_{j}:=\left.\varphi\right|_{S_{j}}$, such that $S_{j}$ is connected. With the previous computations we have

$$
\kappa([C, \varphi])=\sum_{j=1}^{k}\left(\operatorname{ind}_{\alpha}\left(S_{j}, \varphi_{j}\right)+\delta\left(\alpha \smile w\left(S_{j}\right)\right)=\kappa_{\alpha}([C, \varphi])+\delta(\alpha \smile w(C)) .\right.
$$

and the proposition follows. 
We continue with the description of the ,dual“ short exact sequence to (ST). There is a natural group homomorphism $\Omega_{1}^{\mathrm{fr}}(M) \rightarrow \Omega_{1}^{\mathrm{SO}}(M)$, which assigns to every framed 1-submanifold $[C, \varphi]$ the oriented bordism class induced by the orientation framing $\varphi$. This is well-defined since every normally framed bordism in $M$ is also an oriented bordism ( $M$ is oriented). By the seminal work of Thom [18] we have an isomorphism

$$
\Omega_{1}^{\mathrm{SO}}(M) \rightarrow H_{1}(M ; \mathbb{Z})
$$

which assigns every oriented submanifold its fundamental class in $H_{1}(M ; \mathbb{Z})$. Thus we obtain a group homomorphism

$$
\Phi: \Omega_{1}^{\mathrm{fr}}(M) \rightarrow H_{1}(M ; \mathbb{Z})
$$

which is clearly surjective. The kernel of $\Phi$ is at most isomorphic to $\mathbb{Z}_{2}$ and elements of the kernel are represented by framed circles $(S, \varphi)$ such that $S$ is oriented null-bordant, i.e. there is an embedded oriented disc $D \subset M \times I$ with the properties $\partial D=S$ and the orientations of $\partial D$ and $S$ agree. We may equip the normal bundle of $S$ with two framings. If both framings can be extended over $D$ then the kernel is trivial and otherwise $\mathbb{Z}_{2}$.

Lemma 3.12. The restricted degree map $\left.\kappa\right|_{\operatorname{ker} \Phi}: \operatorname{ker} \Phi \rightarrow \Omega_{1}^{f r}$ is an isomorphism.

Proof. Since $\kappa$ is a homomorphism it will map the neutral element of ker $\Phi$ to that of $\Omega_{1}^{\text {fr }}$. Thus it suffices to show the following: Let $(S, \varphi)$ be a framed circle such that $S$ is oriented null-bordant in $M$ but $\varphi$ cannot be extended over the nullbordism. We have to show $\kappa([S, \varphi]) \neq 0$, where 0 denotes the neutral element of $\Omega_{1}^{\mathrm{fr}}$. We may assume that $S$ lies in a chart of $M^{1}$. Thus we may embed $S$ into $\mathbb{R}^{n+1}$ endowed with a normal framing, which cannot be extended over a nullbordism in $\mathbb{R}^{n+1}$. Hence the index of $(S, \varphi)$ defines a non-trivial element in $\Omega_{1}^{\mathrm{fr}}$ (note that since $w(S)=0$ the element $\kappa[(S, \varphi)]$ does not depend on the spin structure of $M$, cf. Lemma 3.11).

Thus we may identify $\operatorname{ker} \Phi$ with $\Omega_{1}^{\text {fr }}$ via $\left(\left.\kappa\right|_{\operatorname{ker} \Phi}\right)^{-1}$ and we obtain a short exact sequence

$$
0 \longrightarrow \Omega_{1}^{\mathrm{fr}} \longrightarrow \Omega_{1}^{\mathrm{fr}}(M) \longrightarrow H_{1}(M ; \mathbb{Z}) \longrightarrow 0
$$

and from Lemma $3.12 \kappa$ is a splitting map. Therefore

Theorem 3.13. Let $M$ be an $(n+1)$-dimensional closed spin manifold. Choose a spin structure on $M$. Then

$$
\Omega_{1}^{f r}(M) \longrightarrow H_{1}(M ; \mathbb{Z}) \oplus \Omega_{1}^{f r}, \quad[C, \varphi] \mapsto([C], \kappa([C, \varphi]))
$$

is an isomorphism of abelian groups.

We finish this section by giving an alternative way to compute the index of a framed circle in the spirit of Pontryagin [14]. Suppose $[S, \varphi]$ is a framed circle, thus there are trivializations of $\nu(S)$ and $\left.T M\right|_{S}$ such that we obtain the stable framing

$$
\varepsilon^{n+1} \cong T S \oplus \varepsilon^{n}
$$

(where is can assume that the isomorphism is orientation preserving). Denote by $v_{1}, \ldots, v_{n+1}$ and by $w_{2}, \ldots, w_{n+1}$ the trivializations of $\left.T M\right|_{S}$ and $\nu(S)$ respectively. Let $w_{1}$ be a trivialization of $T S$. Let $\Phi: T S \oplus \varepsilon^{n} \rightarrow \varepsilon^{n+1}$ be the isomorphism of the stable framing, then there is a matrix $A=\left(A_{i j}\right): S \rightarrow$ $\mathrm{GL}^{+}(n+1)$ (where $\mathrm{GL}^{+}(n+1)$ is the set of all invertible real matrices of size $(n+1) \times(n+1)$ with positive determinant) such that such that

$$
\Phi\left(w_{i}\right)=\sum_{j=1}^{n+1} A_{i j} \cdot v_{j} .
$$

Since $\mathrm{SO}(n+1)$ is a strong deformation retract of $\mathrm{GL}^{+}(n+1)$ we have $\pi_{1}\left(\mathrm{GL}^{+}(n+1)\right) \cong \mathbb{Z}_{2}$. The map $A: S \rightarrow \mathrm{GL}^{+}(n+1)$ defines an element $[A] \in \pi_{1}\left(\mathrm{GL}^{+}(n+1)\right)$. Changing the homotopy classes of trivializations of $\left.T M\right|_{S}$ and $\nu(S)$ does not change $[A]$. Furthermore $[A]$ is also independent of the choice of trivializations of $T S$.

\footnotetext{
${ }^{1}$ Take a small embedded closed disc and choose a framing on the circle bounding the disc which cannot be extended over a proper embedded disc in $M \times I$.
} 
According to the Preliminaries in Section 2 any stable framing ind $(S, \varphi)$ can be represented by a framed circle $S_{0}$ in $\mathbb{R}^{n+1}$ such that

$$
\left.T S_{0} \oplus \varepsilon^{n} \cong T S_{0} \oplus \nu\left(S_{0}\right) \cong\left(T \mathbb{R}^{n+1}\right)\right|_{S_{0}} \cong \varepsilon^{n+1}
$$

recovers the stable framing of $(S, \varphi)$. It follows that

$$
\operatorname{ind}(S, \varphi)=\delta\left(S_{0}, \varphi_{0}\right),
$$

where $\delta$ is the invariant constructed by Pontryagin, [14, Theorem 20]. We will use a different notation: Let us denote by $[A]$ the homotopy class constructed above from the stable framing and by $\overline{[A]}$ the element $[A]+1 \in \Omega_{1}^{\mathrm{fr}}\left(S^{n}\right) \cong \mathbb{Z}_{2}$ where 1 is the non-trivial element. Thus we proved

Lemma 3.14. We identify $\pi_{1}\left(\mathrm{GL}^{+}(n+1)\right)$ with $\Omega_{1}^{f r}$ by the unique isomorphism $\mathbb{Z}_{2} \rightarrow \mathbb{Z}_{2}$. Then

$$
\overline{[A]}=\operatorname{ind}(S, \varphi) .
$$

\section{Computation of $\pi^{n}(M)$}

We start this section to explain the group structure of $\pi^{n}(M)$. Let $j: S^{n} \vee S^{n} \rightarrow S^{n} \times S^{n}$ be the inclusion of the $(2 n-1)$-skeleton of $S^{n} \times S^{n}$ (endowed with the standard CW structure) then, since $M$ is $n+1$-dimensional CW complex, the induced map $j_{\#}:\left[M, S^{n} \vee S^{n}\right] \rightarrow\left[M, S^{n} \times S^{n}\right]$ is an isomorphism. For $f, g \in \pi^{n}(M)$ the group structure is defined by

$$
f+g:=\left(\operatorname{id}_{S^{n}} \vee \operatorname{id}_{S^{n}}\right)_{\#} \circ\left(j_{\#}\right)^{-1}(f \times g) .
$$

This makes $\pi^{n}(M)$ to an abelian group.

Now, let $f: M \rightarrow S^{n}$ be a differentiable map and $x_{0} \in S^{n}$ a regular value. We orient $S^{n}$ by the normal vector field pointing outwards and the standard orientation of $\mathbb{R}^{n+1}$.

Let $\Psi: \pi^{n}(M) \rightarrow H^{n}(M ; \mathbb{Z})$ be the map $\Psi([f]):=f^{*} \sigma$ where $\sigma \in H^{n}\left(S^{n} ; \mathbb{Z}\right)$ is a fixed generator. We define the analogous degree map $\kappa: \pi^{n}(M) \rightarrow \pi_{1}^{S}$, where $\pi_{1}^{S}$ is the first stable homotopy group of spheres, as follows: $\kappa$ is the composition of

$$
\pi^{n}(M) \stackrel{\sim}{\longrightarrow} \Omega_{1}^{\mathrm{fr}}(M) \stackrel{\kappa}{\longrightarrow} \Omega_{1}^{\mathrm{fr}} \stackrel{\sim}{\longrightarrow} \pi_{1}^{S} .
$$

where the first and the last isomorphism is again induced by the Pontryagin-Thom isomorphism.

Theorem 4.1. Let $M$ be a closed $(n+1)$-dimensional spin manifold. Then

(a) The generator of $\operatorname{ker} \Psi \cong \mathbb{Z}_{2}$ is given by the homotopy class of the map $\eta \circ \omega: M \rightarrow S^{n+1}$, where $\eta$ represents a generator of $\pi_{n+1}\left(S^{n}\right)$ and $\omega: M \rightarrow S^{n+1}$ is a map of odd degree. Thus $\operatorname{ker} \Psi \cong \pi_{n+1}\left(S^{n}\right)$.

(b) Identifying $\pi_{1}^{S}$ with $\pi_{n+1}\left(S^{n}\right)$ the degree map $\kappa: \pi^{n}(M) \rightarrow \pi_{1}^{S}$ splits the short exact sequence (ST). Thus we have

$$
\pi^{n}(M) \longrightarrow H^{n}(M ; \mathbb{Z}) \oplus \pi_{n+1}\left(S^{n}\right), \quad[f] \mapsto\left(f^{*} \sigma, \kappa([f])\right) .
$$

is an isomorphism of abelian groups.

Proof. Clearly we have $[\eta \circ \omega] \in \operatorname{ker} \Psi$. For (a) it is enough to check that $\kappa([\eta \circ \omega])$ is the zero in $\pi_{1}^{S}$. We choose an odd degree map $\omega: M \rightarrow S^{n+1}$ as follows: Let $\left\{p_{1}, \ldots, p_{l}\right\}$ be the preimage of a regular value $y_{0}$ and choose open sets $U_{1}, \ldots, U_{l} \subset M$ as well as $V \subset S^{n+1}$ such that for all $i=1, \ldots, l$

(a) $U_{i}$ and $V$ are contractible,

(b) $p_{i} \in U_{i}$ and $y_{0} \in V$,

(c) there are charts $\psi_{i}: U_{i} \rightarrow \mathbb{R}^{n+1}, \psi: V \rightarrow \mathbb{R}^{n+1}$,

(d) $\omega_{i}:=\left.\omega\right|_{U_{i}}$ is an orientation preserving diffeomorphism onto $V$. 
Since $p$ has odd degree, $l$ has to be an odd number (such maps exists e.g. using the Pontryagin-Thom construction). Furthermore let $x_{0} \in S^{n}$ be a regular value of $\eta$ and $S_{0}=\eta^{-1}\left(x_{0}\right)$. We may assume that $S_{0}$ is connected (e.g. see [13, Theorem C]) and $S_{0} \subset V$. Let $\varphi_{0}$ be the framing of $\nu\left(S_{0}\right)$ induced by $\eta$, then $0 \neq\left[S_{0}, \varphi_{0}\right] \in \Omega_{1}^{\text {fr }}\left(S^{n+1}\right) \cong \pi_{n+1}\left(S^{n}\right) \cong \mathbb{Z}_{2}$ and therefore by definition we have ind $\left(S_{0}, \varphi_{0}\right) \neq 0$.

Denote by $S_{i}:=\omega_{i}^{-1}\left(S_{0}\right)$ and frame $\nu\left(S_{i}\right)$ by $\varphi_{0}$ and $d \omega_{i}$. Then $C=S_{1} \cup \ldots \cup S_{l}$ together with the framings $\varphi_{i}$ is a Pontryagin manifold for $\eta \circ \omega$ to the regular value $x_{0}$. Note that $w\left(S_{i}\right)=0$ for $i=1, \ldots, l$, since they are contained in a chart of $M$. By Proposition 3.11 this means that their indices do not depend on the spin structure of $M$. Clearly we deduce $\operatorname{ind}\left(S_{i}, \varphi_{i}\right)=\operatorname{ind}\left(S_{0}, \varphi_{0}\right) \neq 0$ for all $i=1, \ldots, l$ and from that we infer

$$
\kappa([\eta \circ \omega])=\sum_{i=1}^{l} \operatorname{ind}\left(S_{i}, \varphi_{i}\right)=l \cdot \operatorname{ind}\left(S_{0}, \varphi_{0}\right) \neq 0
$$

since $l$ is odd, which proves (a).

Part (b) follows directly from part (a).

Corollary 4.2. Suppose $M$ is simply connected, then, up to homotopy, there are exactly two maps $M \rightarrow S^{n}$ and one of them is the constant map. The homotopy class of the non-trivial map is represented by $\eta \circ \omega: M \rightarrow S^{n}$, see Theorem 4.1.

Finally we would like to show, that $\kappa$ is natural with respect to maps between manifolds which preserve the spin structure

Proposition 4.3. Suppose $\Phi: M_{1} \rightarrow M_{2}$ is a map between two closed and connected spin manifolds of dimension $(n+1)$. We assume that the spin structure of $M_{1}$ coincides with the pull-back spin structure by $\Phi$ of $M_{2}$. Then for the natural homomorphism $\Phi^{\#}: \pi^{n}\left(M_{2}\right) \rightarrow \pi^{n}\left(M_{1}\right), f \mapsto \Phi \circ f$ we have

$$
\kappa\left(\Phi^{\#}(f)\right)=\operatorname{deg}_{2} \Phi \cdot \kappa(f) .
$$

where $\operatorname{deg}_{2} \Phi$ is the mod 2 degree of $\Phi$. Therefore using the isomorphism

$$
\pi^{n}(M) \cong H^{n}(M ; \mathbb{Z}) \oplus \pi_{n+1}\left(S^{n}\right)
$$

we have

$$
\Phi^{\#}: \pi^{n}\left(M_{2}\right) \rightarrow \pi^{n}\left(M_{1}\right), \quad(\alpha, \nu) \mapsto\left(\Phi^{*}(\alpha), \operatorname{deg}_{2} \Phi \cdot \nu\right)
$$

Proof. First note that $\Phi^{\#}$ is well-defined on the homotopy class of $\Phi$. For $f \in \pi^{n}\left(M_{2}\right)$ there is a decomposition $f=f_{\alpha}+f_{\nu}$ with $\kappa\left(f_{\alpha}\right)=0, f_{\alpha}^{*}(\sigma)=\alpha$ and $\kappa\left(f_{\nu}\right)=\nu$ as well as $f_{\nu}^{*}(\sigma)=0$.

Let us show first $\Phi^{\#}\left(f_{\alpha}\right)=f_{\Phi^{*}(\alpha)}$. Clearly we have $\Phi^{\#}\left(f_{\alpha}\right)(\sigma)=\Phi^{*}(\alpha)$ thus it remains to show $\kappa\left(\Phi^{\#}\left(f_{\alpha}\right)\right)=0$. Let $C_{2}$ be the preimage of a regular value of $f_{\alpha}$ with a normal framing $\varphi_{0}$ such that $\kappa\left(\left[C_{2}, \varphi_{0}\right]\right)=0$. Moreover we may choose $f_{\alpha}$ such that each framed circle of $\left(C_{2}, \varphi_{0}\right)$ has index 0 . Deform $\Phi$ to be transversal to $C_{2}$, thus $C_{1}:=\Phi^{-1}\left(C_{2}\right)$ is a closed 1-dimensional submanifold of $M_{1}$. The normal bundle to $C_{1}$ is isomorphic to the pull back of the normal bundle of $C_{2}$ by $\Phi$. This induces a framing on $C_{2}$ such that every framed circle thereof has index 0 (note that the spin structure of $M_{1}$ is the pulled back by $\Phi$ from $M_{2}$ ) which is also the framing induced by the map $f_{\alpha} \circ \Phi$. But this means $\kappa\left(\Phi^{\#}\left(f_{\alpha}\right)\right)=0$.

On the other hand we may assume a preimage of a regular point in $S^{n}$ under $f_{\nu}$ is a contractible circle $S_{2}$ in $M_{2}$ with normal framing $\varphi$ such that the index of the framed circle $\left(S_{2}, \varphi\right)$ is $\nu \in \pi_{n+1}\left(S^{n}\right)$. Then making again $\Phi$ transverse to $S_{2}$ we obtain a normally framed submanifold $\left(C_{1}, \varphi\right)$ such that the index of each framed circle in $C_{1}$ has index $\nu$. As in the proof of Theorem 4.1 the degree of $\left(C_{1}, \varphi\right)$ is just $\operatorname{deg}_{2} \Phi \cdot \nu$. Therefore $\Phi^{\#}\left(f_{\nu}\right)=f_{\operatorname{deg}_{2} \Phi \cdot \nu}$ and the proposition follows.

Corollary 4.4. Let $f: M \rightarrow S^{n}$ and $x_{0} \in S^{n}$ a regular value. Write $S_{1} \cup \ldots \cup S_{k}=f^{-1}\left(x_{0}\right)$ such that $S_{i}$ is a connected component of $f^{-1}\left(x_{0}\right)$ and denote by $\varphi_{i}$ the induced framing from $f$. Then the number

$$
\#\left\{i: \kappa\left(\left[S_{i}, \varphi_{i}\right]\right) \neq 0\right\} \bmod 2
$$

does not depend on $x_{0}$ and is a homotopy invariant. 


\section{Application to vector bundles}

In this section $\pi: E \rightarrow M$ should denote an oriented vector bundle of rank $n$ endowed with a spin structure. Let $s: M \rightarrow M$ be a section. If not otherwise stated, we say $s$ is transversal if $s$ is transversal to the zero section $0_{E}$ of $E$. For a transversal section $s$ the zero locus $C$ is a smooth 1-dimensional closed submanifold of $M$. The differential $d s: T M \rightarrow T E$ restricted to $\nu(C)$ is an isomorphism of the vector bundles $\left.\nu(C) \rightarrow E\right|_{C}$. Since $E$ possess a spin structure, by Lemma $\left.3.1 E\right|_{C}$ has a framing and with $d s$ this endows $\nu(C)$ with the framing $\varphi$ of $\left.E\right|_{C}$. Note that the homology class $[C] \in H_{1}(M ; \mathbb{Z})$ is the Poincaré dual of the Euler class of $E$.

Proposition 5.1. The class $[C, \varphi] \in \Omega_{1}^{f r}(M)$ does not depend on the section s.

Proof. Let $s^{\prime}: M \rightarrow E$ be another transversal section and denote the corresponding normally framed zero locus by $\left(C^{\prime}, \varphi^{\prime}\right)$. Let $s^{*}: M \times I \rightarrow \operatorname{pr}^{*}(E)$ be a section of $\operatorname{pr}^{*}(E) \rightarrow M \times I$ (where pr: $M \times I \rightarrow M$ ) such that $\left.s^{*}\right|_{M \times 0}=s$ and $\left.s^{*}\right|_{M \times 1}=s^{\prime}$. There we may deform $s^{*}$ to a section $\hat{s}$ which is transverse to the zero section of $\operatorname{pr}^{*}(E) \rightarrow M \times I$ and agrees with $s$ and $s^{\prime}$ on the boundary of $M \times I$. The zero locus of $\hat{s}$, call it $\Sigma \subset M \times I$ is a bordism between $C$ and $C^{\prime}$ by construction. Moreover by Lemma $\left.3.1 T(M \times I)\right|_{\Sigma}$ inherits a framing from the spin structure of $M$ as well as $\nu(\Sigma)$ from $d \hat{s}$ and the spin structure of $\left.\operatorname{pr}^{*}(E)\right|_{\Sigma}$. Thus $\Sigma$ is a normally framed bordism between $(C, \varphi)$ and $\left(C^{\prime}, \varphi^{\prime}\right)$.

Definition 5.2. The bordism class $[C, \varphi] \in \Omega_{1}^{\mathrm{fr}}(M)$ constructed above is called the framed divisor of $E \rightarrow M$. Furthermore we define the degree $\kappa(E)$ of $E$ as $\kappa([C, \varphi])$

For $[C, \varphi] \in \Omega_{1}^{\mathrm{fr}}(M)$ we denoted by $w(C) \in H^{n}\left(M ; \mathbb{Z}_{2}\right)$ the Poincaré dual of the $\mathbb{Z}_{2}$ fundamental class $[C] \in H_{1}\left(M ; \mathbb{Z}_{2}\right)$. If $[C, \varphi]$ is the framed divisor of $E \rightarrow M$ then $w(C)$ is the $n$-th Stiefel-Whitney class $w_{n}(E)$ (since $w_{n}(E)$ is the Euler class $e(E)$ modulo 2). Therefore if $w_{n}(E)=0$ then the degree of $E$ does not depend on the spin structure (see Lemma 3.1 and Proposition 3.11).

Proposition 5.3. If $w_{n}(E)=0$ then the framed divisor is independent of the spin structures on $M$ and E.

For the next theorem we will need a technical Lemma. Let $D^{m}$ denote the closed unit ball in $\mathbb{R}^{m}$ and consider a smooth map $f: D^{n+k+1} \rightarrow \mathbb{R}^{n+1}$. Assume that $0 \in \mathbb{R}^{n+1}$ is a regular value for $f$ and $\Sigma_{f}^{k}:=f^{-1}(0)$ does not intersect the boundary of $D^{n+k+1}$. Denote by $\varphi_{f}$ the induced framing on $\nu\left(\Sigma_{f}^{k}\right)$. Since $\Sigma_{f}^{k}$ is a submanifold of $\mathbb{R}^{n+k+1}$ the trivialization $\varphi_{f}$ defines a stable tangential framing of $\Sigma_{f}^{k}$ thus the pair $\left(\Sigma_{f}^{k}, \varphi_{f}\right)$ defines an element in $\Omega_{k}^{\mathrm{fr}}$. On the other side, consider

$$
g: S^{n+k}=\partial D^{n+k+1} \rightarrow S^{n}, \quad g(x):=\frac{f(x)}{|f(x)|}
$$

and choose a regular value $y \in S^{n}$. Denote by $\left(\Sigma_{g}^{k}, \varphi_{g}\right)$ the induced stably framed manifold.

Lemma 5.4. With the notation above we have that $\left(\Sigma_{f}^{k}, \varphi_{f}\right)$ and $\left(\Sigma_{g}^{k}, \varphi_{g}\right)$ are stably framed bordant, thus they define the same element in $\Omega_{k}^{f r}$.

Proof. There is an $\varepsilon>0$ such that the closed ball $D_{\varepsilon}$ centered in $0 \in \mathbb{R}^{n+1}$ with radius $\varepsilon$ contains only regular values of $f$. The preimage of $D_{\varepsilon}$ under $f$ is a disc bundle $D\left(\Sigma_{f}^{k}\right)$ of the normal bundle $\nu\left(\Sigma_{f}^{k} \hookrightarrow \mathbb{R}^{n+k+1}\right)$. Denote by $S\left(\Sigma_{f}^{k}\right)$ its sphere bundle. Then $\left.f\right|_{S\left(\Sigma_{f}^{k}\right)}$ has image $S_{\varepsilon}=\partial D_{\varepsilon}$. Thus for $y^{\prime} \in S_{\varepsilon}, \Sigma_{y^{\prime}}=\left(\left.f\right|_{S\left(\Sigma_{f}^{k}\right)}\right)^{-1}\left(y^{\prime}\right)$ lies completely in $S\left(\Sigma_{f}^{k}\right)$. Moreover the Pontryagin manifold $\left(\Sigma_{y^{\prime}}, \varphi_{y^{\prime}}\right)$ is framed bordant to $\left(\Sigma_{f}, \varphi_{f}\right)$. Thus we would like to show that $\left(\Sigma_{y^{\prime}}, \varphi_{y^{\prime}}\right)$ represents the same element in $\Omega_{k}^{\mathrm{fr}}$ as $\left(\Sigma_{g}, \varphi_{g}\right)$. Since the normal bundle of $S\left(\Sigma_{f}^{k}\right)$ is trivial the framing $\varphi_{y^{\prime}}$ induces a framing $\varphi_{y^{\prime}}^{\prime}$ on $\nu\left(\Sigma_{y^{\prime}} \hookrightarrow S\left(\Sigma_{f}^{k}\right)\right)$ such that $\left(\Sigma_{y^{\prime}}, \varphi_{y^{\prime}}\right)$ is stably framed bordant to $\left(\Sigma_{y^{\prime}}, \varphi_{y^{\prime}}^{\prime}\right)$. But the latter normally framed manifold is the Pontryagin manifold to the map $\left.f\right|_{S\left(\Sigma_{f}^{k}\right)}: S\left(\Sigma_{f}^{k}\right) \rightarrow S_{\varepsilon}$ at the point $y^{\prime} \in S_{\varepsilon}$.

Let $N$ be the complement of the interior of $D\left(\Sigma_{f}^{k}\right)$ in $D^{n+k+1}$. Then $N$ is a framed cobordism between $S^{n+k}=\partial D^{n+k+1}$ and $S\left(\Sigma_{f}^{k}\right)$. The restriction of the map

$$
F: N \rightarrow S^{n}, \quad F(x):=\frac{f(x)}{|f(x)|}
$$


to $S^{n+k}$ is equal to $g$ and $F$ restricted to $S\left(\Sigma^{k}\right)$ is equal to $\varepsilon^{-1} \hat{f}$. Hence $F$ defines a framed bordism between $\left(\Sigma_{g}^{k}, \varphi_{g}\right)$ and $\left(\Sigma_{y^{\prime}}, \varphi_{y^{\prime}}^{\prime}\right)$ which proves the lemma.

Theorem 5.5. Let $E \rightarrow M$ be an oriented vector bundle of rank $n$ with $w_{2}(E)=0$ over a closed spin manifold $M$ of dimension $n+1$. Then $E$ admits a nowhere vanishing section if and only if the Euler class is zero and $\kappa(E)=0$.

Proof. Suppose there is a nowhere vanishing section of $E$ then clearly this section is transverse and has an empty framed divisor. Thus from Theorem 3.13 we have that the Euler class must be zero and $\kappa(E)=0$.

Assume now that $e(E)=0$ and $\kappa(E)=0$. Consider the fibration

$$
S^{n-1} \longrightarrow B \mathrm{SO}(n-1) \longrightarrow B \mathrm{SO}(n)
$$

where $B \mathrm{SO}(k)$ denotes the classifying space to the special orthogonal group $\mathrm{SO}(k)$. Consider the classifying map $g: M \rightarrow B S O(n)$ for $E \rightarrow M$. There exists a nowhere vanishing section if and only if there is a lift $\hat{g}: M \rightarrow B \mathrm{SO}(n-1)$ of $g$ up to homotopy.

First we put a CW-structure on $M$ (e.g. induced by a Morse function) then over the $(n-2)$-skeleton of $M$ there exists such a lift $\hat{g}$ of $g$. The obstruction to extend the lift over the $n$-skeleton lies in $H^{n}\left(M ; \pi_{n-1}\left(S^{n-1}\right)\right)=H^{n}(M ; \mathbb{Z})$ which is given by the Euler class $e(E)$. Since this is assumed to be zero $\hat{g}$ extends over the $n$-skeleton of $M$. The obstruction to extend $\hat{g}$ over the top cell of $M$ lies in $H^{n+1}\left(M ; \pi_{n}\left(S^{n-1}\right)\right) \cong \pi_{n}\left(S^{n-1}\right) \cong \mathbb{Z}_{2}$. Let $e_{n+1}$ be the top cell of $M$ and $\psi: \partial e_{n+1} \cong S^{n} \rightarrow M$ the corresponding attaching map. The bundle $\left.E\right|_{e_{n+1}}$ is canonical isomorphic to $e_{n+1} \times \mathbb{R}^{n}$. Let $\sigma: M \rightarrow E$ be a section which has no zeroes over the $n$-skeleton of $M$ and which is transverse to the zero section of $E$. Then consider the map

$$
g: \partial e_{n+1} \cong S^{n} \rightarrow S^{n-1}, \quad g(x):=\frac{\sigma \circ \psi(x)}{|\sigma \circ \psi(x)|}
$$

(where the norm is take with respect to a euclidean bundle metric on $E$ ). The homotopy class of $g$ in $\pi_{n}\left(S^{n-1}\right)$ is the obstruction to extend a no where vanishing section over the $n$-skeleton to the $(n+1)$ skeleton of $M$. Since $\pi_{n}\left(S^{n-1}\right)$ is isomorphic to the stable homotopy group $\pi_{1}^{S}$ we consider the homotopy class of $g$ as an element therein.

From Lemma 5.4 we infer that the $[g] \in \pi_{1}^{S} \cong \Omega_{1}^{\text {fr }}$ is equal to the framed divisor $\kappa(E)$ of $E$ defined by $\sigma$, thus $E$ admits a no where vanishing section in case $e(E)=0$ and $\kappa(E)=0$.

Example 5.6. As an application of our theory we will reprove the following fact due to Whitehead [20] and Eckmann [5]: The number of linear independent vector fields on $S^{4 k+1}$ is equal to 1 (see also [1] and in [19]).

Denote by $\langle\cdot, \cdot\rangle$ the standard euclidean product in $\mathbb{R}^{4 k+2}$. The vector field

$$
v: \mathbb{R}^{4 k+2} \rightarrow \mathbb{R}^{4 k+2}, \quad v\left(x_{1}, x_{2}, \ldots, x_{4 k+2}\right)=\left(-x_{2}, x_{1}, \ldots,-x_{4 k+2}, x_{4 k+1}\right)
$$

defines a nowhere vanishing vector field on $S^{4 k+1}$ since $\langle v(x), x\rangle=0$ for $x \in S^{4 k+1}$. Let $E$ the subbundle of $T S^{4 k+1}$ orthogonal to the line bundle spanned by $v$. For any vector field on $S^{4 k+1}$ which is in every point linear independent to $v$ there is a nowhere vanishing section of $E^{2}$. Since the Euler class of $E$ vanishes, it suffices to show that $\kappa(E)$ is not zero by Theorem 5.5 (note that the spin structures of $S^{4 k+1}$ and that of $E$ are unique up to homotopy).

Consider now the vector field

$$
w: \mathbb{R}^{4 k+2} \rightarrow \mathbb{R}^{4 k+2}, \quad w(x)=\left(0,0,-x_{5}, x_{6}, x_{3},-x_{4},-x_{9}, x_{10}, x_{7},-x_{8}, \ldots\right)
$$

Since $\langle w(x), x\rangle=\langle w(x), v(x)\rangle=0$ we have that $w$ is a section of $E$. Furthermore $w$ is transverse to the zero section of $E$ and the zero locus is given by

$$
S=\left\{\left(x_{1}, x_{2}, 0, \ldots, 0\right) \in S^{4 k+1}: x_{1}^{2}+x_{2}^{2}=1\right\} .
$$

\footnotetext{
${ }^{2}$ For any pair on orthonormal vector fields $v_{1}, v_{2}$ of $S^{4 k+1}$ one can choose a new pair of orthonormal vector fields which consists of $v$ and an section of $E$.
} 
In Example 3.10 we saw that $\left.T S^{4 k+1}\right|_{S}$ inherits the standard framing from the spin structure. But the induced framing on $\left.E\right|_{S}$ cannot be the standard framing. To see this assume it inherits the standard framing and let $\tau_{1}, \ldots, \tau_{n}$ be a trivialization of $\left.E\right|_{S}$, then, since the spin structure on $E$ is induced by $T S^{4 k+1}$ and $v$, the map $S \rightarrow \mathrm{SO}(4 k+2), x \mapsto\left(x, v(x), \tau_{1}(x), \ldots, \tau_{n}(x)\right)$ has to be nullhomotopic cf. Example 3.10 (note that $\left.v\right|_{S}$ is tangent to $S$ ) which is a contradiction. Thus from Example 3.10 we deduce that the index of the framed divisor is not zero, hence $\kappa(E)=1$ and therefore $E$ does not admit a nowhere vanishing section from Theorem 5.5.

Remark 5.7. In [6, Theorem 1.6] the authors show, that for any $n$-dimensional CW-complex of dimension $X$ and any $k$-dimensional integral cohomology class $a \in H^{k}(X ; \mathbb{Z})$ there exists an oriented vector bundle over $X$ whose Euler class equals $2 \cdot N(n, k) \cdot a$.

Suppose $\operatorname{dim} X=2 k+1$. By Steenrod's exact sequence (ST) it follows that the Hurewicz map $\pi^{n}(X) \rightarrow H^{n}(X ; \mathbb{Z})$ is surjective. Then for every $a \in H^{n}(X ; \mathbb{Z})$ there is a map $f_{a} \in \pi^{n}(X)$ such that $f_{a}^{*}(\sigma)=a$, where $\sigma \in H^{n}(S ; \mathbb{Z})$ denotes the generator such that $2 \sigma$ equals to the Euler class of the tangent bundle $T S^{n}$ of $S^{n}$. Clearly the vector bundle $f_{a}^{*}\left(T S^{n}\right)$ has Euler class $2 \cdot a$ and therefore $N(2 k, 2 k+1)=1$ in the notation of [6].

Note that any vector bundle over $S^{n}$ for $n \neq 2,4,8$ has an Euler class divisible by 2, cf. [2,11]. In the cases $n=2,4,8$ there are real vector bundles whose Euler class is a generator of $H^{n}\left(S^{n} ; \mathbb{Z}\right)$, namely the associated bundles to the Hopf fibrations $S^{2 n-1} \rightarrow S^{n}$. We deduce

Proposition 5.8. Suppose $n=4$ or $n=8$ and let $M$ be a $(n+1)$-dimensional closed spin manifold. Denote by $\operatorname{Vect}_{n}(M)$ the set oriented vector bundles over $M$ of rank $n$ up to isomorphism. Let $E_{0} \rightarrow S^{n}$ denote the oriented rank $n$ vector bundle such that the Euler class of $E_{0}$ is a generator of $H^{n}\left(S^{n} ; \mathbb{Z}\right)$. Then the map

$$
\pi^{n}(M) \rightarrow \operatorname{Vect}_{n}(M), \quad f \mapsto f^{*}\left(E_{0}\right)
$$

is injective.

Proof. We consider $f_{1}, f_{2} \in \pi^{n}(M)$ such that $E_{1}:=f_{1}^{*}\left(E_{0}\right) \cong f_{2}^{*}\left(E_{0}\right)=: E_{2}$ since they represent the Euler class the respective bundles. This implies $f_{1}^{*}(\sigma)=f_{2}^{*}(\sigma)$ for a generator in $H^{n}\left(S^{n} ; \mathbb{Z}\right)$. Thus it remains to show that $\kappa\left(f_{1}\right)=\kappa\left(f_{2}\right)$. Let $x_{i} \in S^{n}$ be a regular value for $f_{i}$ for $i=1,2$. There is a section $\sigma_{0, i}: S^{n} \rightarrow E_{0}$ which is transverse to the zero section with an isolated zero in $x_{i}$ (note that the Poincaré dual of $x_{i}$ in $S^{n}$ represents the Euler class of $E_{0}$. Therefore $\sigma_{0, i}$ can only exist since if the Euler class is a generator, since the index of transverse sections is always \pm 1$)$. Then $\sigma_{i}:=f^{*}\left(\sigma_{0, i}\right)$ is a transverse section of $E_{i}$. Note that from the Pontryagin-Thom construction we may assume that $f_{i}^{-1}\left(x_{i}\right)$ is connected, hence the zero locus of $\sigma_{i}$ coincides with $f_{i}^{-1}\left(x_{i}\right)$. Moreover the framed divisor of $E_{i}$ coincides with the degree of $f_{i}$ (cf. Definitions 3.8 and 5.2). Since $E_{1} \cong E_{2}$ we have $\kappa\left(E_{1}\right) \cong \kappa\left(E_{2}\right)$ by construction of the framed divisor and Proposition 5.1. From $f_{1}^{*}(\sigma)=f_{2}^{*}(\sigma)$ and $\kappa\left(f_{1}\right)=\kappa\left(E_{1}\right)=\kappa\left(E_{2}\right)=\kappa\left(f_{2}\right)$ it follows from Theorem 4.1 that $f_{1}$ is homotopic to $f_{2}$.

\section{References}

[1] Adams, J. F. Vector fields on spheres. Bull. Amer. Math. Soc. 68 (1962), 39-41.

[2] Atiyah, M. F., And Hirzebruch, F. Bott periodicity and the parallelizability of the spheres. Proc. Cambridge Philos. Soc. 57 (1961), 223-226.

[3] Borsuk, K. Sur un espace des transformations continues et ses applications topologiques. Monatsh. Math. Phys. 38, 1 (1931), 381-386.

[4] Davis, J. F., AND KIRK, P. Lecture notes in algebraic topology, vol. 35 of Graduate Studies in Mathematics. American Mathematical Society, Providence, RI, 2001.

[5] Eckmann, B. Systeme von Richtungsfeldern in Sphären und stetige Lösungen komplexer linearer Gleichungen. Comment. Math. Helv. 15 (1943), 1-26.

[6] Guijarro, L., Schick, T., and Walschap, G. Bundles with spherical Euler class. Pacific J. Math. 207, 2 (2002), 377-391. 
[7] Kirby, R., Melvin, P., And Teichner, P. Cohomotopy sets of 4-manifolds. In Proceedings of the Freedman Fest (2012), vol. 18 of Geom. Topol. Monogr., Geom. Topol. Publ., Coventry, pp. 161-190.

[8] Konstantis, P. Deforming a section to a section without zeros. MathOverflow.

[9] Konstantis, P. Vector bundles and cohomotopies of spin 5-manifolds.

[10] Larmore, L. L., And Thomas, E. Group extensions and principal fibrations. Math. Scand. 30 (1972), 227-248.

[11] Milnor, J. Some consequences of a theorem of Bott. Ann. of Math. (2) 68 (1958), 444-449.

[12] Milnor, J. Spin structures on manifolds. Enseignement Math. (2) 9 (1963), 198-203.

[13] Milnor, J. W. Topology from the differentiable viewpoint. Based on notes by David W. Weaver. The University Press of Virginia, Charlottesville, Va., 1965.

[14] Pontryagin, L. S. Smooth manifolds and their applications in homotopy theory. In American Mathematical Society Translations, Ser. 2, Vol. 11. American Mathematical Society, Providence, R.I., 1959, pp. 1-114.

[15] Spanier, E. Borsuk's cohomotopy groups. Ann. of Math. (2) 50 (1949), 203-245.

[16] Steenrod, N. E. Products of cocycles and extensions of mappings. Ann. of Math. (2) 48 (1947), 290-320.

[17] TAYlor, L. R. The principal fibration sequence and the second cohomotopy set. In Proceedings of the Freedman Fest (2012), vol. 18 of Geom. Topol. Monogr., Geom. Topol. Publ., Coventry, pp. $235-251$.

[18] Тном, R. Quelques propriétés globales des variétés différentiables. Comment. Math. Helv. 28 (1954), 17-86.

[19] Thomas, E. The index of a tangent 2-field. Comment. Math. Helv. 42 (1967), 86-110.

[20] Whitehead, G. W. Homotopy properties of the real orthogonal groups. Ann. of Math. (2) 43 (1942), 132-146.

[21] Wu, W.-T. Classes caractéristiques et i-carrés d'une variété. C. R. Acad. Sci. Paris 230 (1950), 508-511.

Panagiotis Konstantis, Department Mathematik/Informatik, Abteilung Mathematik, Universitt zu Kln, Weyertal 86-90, $50931 \mathrm{Kln}$, Germary, E-mail address: pako@math.uni-koeln.de 